\title{
Disruption of bradycardia associated with discriminative conditioning in combat veterans with PTSD
}

\author{
Jay $\mathrm{P}$ Ginsberg ${ }^{1,2}$ \\ Edwin Ayers ${ }^{3}$ \\ Louisa Burriss' \\ Donald A Powell 1,4 \\ 'Shirley L. Buchanan Neuroscience \\ Laboratory, Dorn VA Medical Center, \\ Columbia, SC, USA; ${ }^{2}$ Department \\ of Pharmacology, Physiology, and \\ Neuroscience, School of Medicine, \\ ${ }^{4}$ Department of Psychology, University \\ of South Carolina, Columbia, SC, USA; \\ ${ }^{3}$ Department of Psychology, South \\ Carolina State University, Orangeburg, \\ SC, USA
}

\begin{abstract}
The effects of combat-related posttraumatic stress disorder (PTSD) on heart rate (HR) responding associated with a discriminative delay eyeblink (EB) conditioning paradigm are reported. Combat PTSD+, Combat PTSD-, and Noncombat PTSD- veterans were assessed with psychometric self-report measures, and baseline heart rate variability (HRV) was measured before receiving a 72-trial session of discriminative EB classical conditioning. Two types (red or green light) of conditioned stimuli (CS) were used: one (CS+) predicted a tone, followed immediately by an aversive stimulus (corneal airpuff); the other (CS-) predicted a tone alone, not followed by the airpuff. The light signal was presented for 5 seconds, during which HR was measured. On all psychometric measures, the PTSD+ subgroup was significantly different from the PTSD- subgroups (Combat + Noncombat), and the PTSD- subgroups did not significantly differ from each other. A linear deceleration in HR to CS+ and CS- signals was found in the combined PTSD- subgroup and on CS- trials in the PTSD+ subgroup, but was not present on $\mathrm{CS}+$ trials in the PTSD+ subgroup. Results are interpreted with respect to a behavioral stages model of conditioned bradycardia and in terms of neural substrates which are both critical to HR conditioning and known to be abnormal in PTSD.
\end{abstract}

Keywords: bradycardia, PTSD, combat veterans, classical conditioning

\section{Introduction}

Posttraumatic stress disorder (PTSD) significantly reduces associative learning of the somatomotor eyeblink (EB) response in Combat PTSD+ veterans in simple (Ayers et al 2003) and discriminative (Ginsberg et al 2007) delay classical (Pavlovian) conditioning. The latter study was designed to yield concomitant EB and heart rate (HR) discriminative conditioning. The characteristics of the EB data are published in full elsewhere (Ginsberg et al 2007), but in brief, EB conditioned response (CR) amplitude was significantly greater for the conditioned stimuli (CS) CS+ (a tone, followed immediately by an aversive stimulus [corneal airpuff]) than to CS- (a tone alone, not followed by the airpuff) for all three groups, and EB amplitude to both the unconditioned stimuli (US; airpuff) and the CS+ declined over trials and was significantly lower in the combat PTSD+ group compared with the combined control goups. The present study examines the HR changes that accompanied the reduced EB responding in PTSD+ combat veterans in that discriminative delay classical conditioning paradigm.

When classical conditioning contingencies elicit a specific somatomotor response, such as an EB CR, nonspecific learned responses of autonomic function, such as HR and skin conductance, are also elicited. Nonspecific responses occur regardless of the nature of the conditioning contingency (Powell et al 1990). For example, signaled electric shock USs delivered to either the orbital region or foot pad of animals result in nonspecific autonomic $\mathrm{CRs}$ that are quite similar regardless of the site of application 
of the US, while the learned somatomotor response is specific to the US - EB in the former case and leg flexion in the latter. Furthermore, the parametric features of classically conditioned nonspecific autonomic responses are very different from those of somatomotor responses (Powell et al 2000), most notably that associatively learned autonomic responses are acquired when the interstimulus interval (ISI) is long (4-8 seconds vs. 0.5 seconds or less for somatomotor learning) and in fewer trials (10 or less vs. 50 or more for somatomotor responses).

Different processes occur during the longer ISI supporting acquisition of conditioned responding of autonomic functions such as HR. An initial short-duration component is referred to as the "registration" or "orienting" component (Maxwell et al 1994), which involves a response to novel stimulation (the orienting response; OR). The normal OR, which has been recognized for some time (Ohman 1988), is a well-studied complex of autonomic changes (including HR deceleration) which occurs in response to novel stimulation that is neutral in valence (a potential CS). Unreinforced novel stimuli elicit stimulus-specific nonsignal ORs that habituate. Habituation, or reduction in strength of a reflexive response produced by repeated presentation of an eliciting unreinforced stimulus, occurs as novelty of the stimulus features wanes due to lack of adaptive significance.

Reinforced stimuli, however, begin to elicit a signal OR. That is, if the signal has significance, it will continue to be attended to and elicit a response. The signal OR thus is part of the process of establishing a CR. It does not habituate and is a component of the CS that results from learning that properties of the reinforcing US are associated with the eliciting stimulus. Thus, if pairing of a potential CS and some type of US reinforcement occurs, a "significance code" is formed indicating that the CS has a contingency with the US. This is the "contingency" component of the process of acquisition of conditioned autonomic responding (Maxwell et al 1994). At this point, nonspecific autonomic CRs appear, including a sustained monotonic deceleration of HR. Thus, the process of acquisition of conditioned HR deceleration comprises the OR and this second stage, is termed as "contingent bradycardia." Together, the signal OR and contingent bradycardic stages are referred to as "conditioned bradycardia" since they occur when the eliciting stimulus has some adaptive reinforcement. These same two stages correspond to an early idealized representation of the evoked cardiac wave form described by Gatchel and Lang (1973), who defined the first and second decelerative components of HR response (D1 and D2), respectively, as occurring during the first 2 seconds after stimulus onset and the 6 second period subsequent to D1.

These considerations are clearly seen and supported in detail with research data as Stages 1 and 2 of Powell's behavioral stages model of classical (Pavlovian) conditioning, as shown in Figure 1 (Powell 1999, 2007). If appropriate conditioning parameters exist, somatomotor conditioning eventually occurs (stage 3), which is followed by later stages. Conditioned bradycardia, or learned HR deceleration, thus is a generalized, nonspecific, phenomenon of Pavlovian conditioning that occurs regardless of the type of specific somatomotor response that is concomitantly conditioned (Powell 2007).

PTSD is thought to have characteristics of conditioned learning, such that neutral stimuli associated with traumatic events become conditioned stimuli and cause conditioned stress responding (including autonomic systems) (Pitman 1989; Pitman and Delahanty 2005). However, surprisingly, only two experimental studies have actually reported results of conditioned responding in individuals with PTSD (Orr et al 2000; Peri et al 2000). Both of these studies set out specifically to condition autonomic responses in individuals with PTSD, and did not attempt to condition EB somatomotor responses. Both studies reported changes in HR conditional responding associated with PTSD that were attributable to heightened activity of the sympathetic nervous system. The hypothesis of the present study was that evidence of a heightened sympathetic state would also be found in conditioned bradycardia associated with a discriminative delay EB paradigm.

\section{Method}

\section{Participants}

Subjects were selected with the procedure described earlier (Ginsberg et al 2007; Burriss et al 2007). Subjects with any history of major mental illness (schizophrenia, bipolar disorder), active substance abuse, traumatic brain injury (loss of consciousness greater than 15 minutes), or neurological disorder were excluded from participation. Prescribed use of antipsychotic (typical or atypical) or anticonvulsant medication for seizure control was an exclusion criterion.

\section{Background information and military stress}

Personal background information was obtained from the Veterans Information Form (VA Form VIF-1), which records education, marital status, race, occupation, and war era(s) of service. Deployment to a combat zone during active service was verified with documented records from the VA Regional 
Office in Columbia, SC. Assessment of war-zone exposure was made with the combat exposure scale (CES; Keane et al 1989) which has seven items and a total score range of 0-41 based on a differential weighting of individual scale items. A cut-off score of 8 on the CES was used to assign subjects to the combat status; subjects with a CES score of 7 or less were classified as noncombat. In addition, each subject received a structured interview by an experienced clinician (EA) with the Clinician Administered Posttraumatic Stress Scale (CAPS) (Blake et al 2000). The CAPS was used to identify traumatic stress reactions to combat exposure and nonmilitary traumatic events. After the clinical interview, classification into PTSD or non-PTSD was made using a CAPS score of 25 or above, which is classified as mild severity (2000). Subjects who did not meet the criterion for combat exposure (ie, CES equal to or greater than 8) but who did meet the criteria for PTSD were excluded from the analysis.

All co-morbid mental diagnoses in the DVA computerized medical records were recorded. Any problem list or medical record note in the VA medical record indicating a diagnosis of anxiety (other than PTSD), depression, history of (but not active) substance abuse or dependence, psychosis, or personality disorder was tabulated for each subject. The use of both prescribed and nonprescribed drugs was obtained during the initial interview with each subject and verified by checking the patient's medical records. Individual medication agents were coded using the VA National Formulary Drug Classification (see http://www.pbm.va.gov/natform/vaclass.xls).

\section{Psychometric tests}

Subjects all completed the Mississippi Scale of combat-related PTSD (MISS; Keane et al 1988). The Mississippi Scale was used as an indicator of stress-related reactions to active military duty in general because items on it are worded in a way that is not limited to combat trauma (McFall et al 1990; Ginsberg et al 2006). Severity of depression was measured with the self-report Zung Depression Scale (Zung 1965). Anxiety as a mood (state) and personality characteristic (trait) was measured with the self-report Spielberger State-Trait Anxiety Inventory-Form Y (Spielberger et al 1983), which yielded two anxiety scores: State Anxiety and Trait Anxiety.

\section{HRV}

Heart rate spectrum was obtained using the electrocardiogram (ECG) from a 4 minute resting period before the conditioning procedure proper. HR was assessed by standard ECG lead II placements using "Graphics Controls" disposable electrodes. The QRS complex of the ECG was detected by a Coulbourn Schmidt trigger. Interbeat intervals (IBIs) were time-stamped for later analysis of three time periods of interest: 4 minute pre-experiment resting period, 5 second pre-signal baseline period, 4.9 second signal period. IBI were measured in milliseconds, then converted to instantaneous heart rate values (in BPM) by the computer.

Heart rate spectrum analysis was effected using the ECG and integrated respiration rate (RR). HRV occurring at the same frequency of respiration, which is the operational

\begin{tabular}{|c|c|c|c|c|c|c|}
\hline $\begin{array}{l}\text { STAGE OF } \\
\text { LEARNING }\end{array}$ & 1 & 2 & 3 & 4 & 5 & 6 \\
\hline $\begin{array}{l}\text { HYPOTHETICAL } \\
\text { CONSTRUCT }\end{array}$ & $\begin{array}{l}\text { ATTENTION } \\
\text { AND/OR } \\
\text { AROUSAL }\end{array}$ & $\begin{array}{l}\text { SIGNIFICANCE } \\
\text { CODE } \\
\text { FORMATION }\end{array}$ & $\begin{array}{c}\text { REFLEX } \\
\text { FACILITATION }\end{array}$ & $\begin{array}{l}\text { LINKAGE AND } \\
\text { RELEGATION }\end{array}$ & $\begin{array}{l}\text { SUPPRESSION } \\
\text { OFRESPONSE } \\
\text { TO IRRELEVANT } \\
\text { CUES }\end{array}$ & EXTINCTION \\
\hline $\begin{array}{l}\text { BEHAVIORAL } \\
\text { CORRELATES }\end{array}$ & $\begin{array}{l}\text { SOMATIC AND } \\
\text { AUTONOMIC } \\
\text { ORIENTING } \\
\text { RESPONSE }\end{array}$ & $\begin{array}{c}\text { AUTONOMIC } \\
\text { CRs }\end{array}$ & $\begin{array}{c}\text { FIRST } \\
\text { SOMATOMOTOR } \\
\text { CR }\end{array}$ & $\begin{array}{l}\text { ASYMPTOTIC } \\
\text { PERFORMANCE }\end{array}$ & $\begin{array}{l}\text { DIFFERENTIAL } \\
\text { CONDITIONING }\end{array}$ & $\begin{array}{l}\text { DECREASED } \\
\text { RESPONDING }\end{array}$ \\
\hline $\begin{array}{c}\text { BRAIN } \\
\text { CORRELATES }\end{array}$ & HYPOTHALAMUS & $\begin{array}{c}\text { LIMBIC } \\
\text { SYSTEM } \\
\text { STRUCTURES }\end{array}$ & $\begin{array}{l}\text { BASAL } \\
\text { GANGLIA }\end{array}$ & CEREBELLUM & $\begin{array}{l}\text { LIMBIC- } \\
\text { THALAMIC- } \\
\text { STRIATAL- } \\
\text { INTERACTIONS }\end{array}$ & $\begin{array}{l}\text { INFRALIMBIC- } \\
\text { HIPPOCAMPAL- } \\
\text { BRAIN STEM } \\
\text { INTERACTIONS }\end{array}$ \\
\hline
\end{tabular}

Figure I Diagram of a 6-stage model of classical conditioning, showing the hypothetical constructs, behavioral indices, and postulated neuroanatomical structures/mechanisms involved in each stage. The degree of experimental support for the latter varies depending upon the stage involved, but substantial evidence exists implicating the indicated structures in the behaviors listed in each case. Although the model suggests sequential processing in a step wise order, as noted in the text, some types of processing are almost certainly simultaneous and take place in a parallel distributed fashion (Powell 2007). 
definition of RSA, was measured in the time-domain by computing HPSSD, defined as the smoothed standard deviation of the heart period interbeat interval over the $4 \mathrm{~min}$ resting period (Richards and Casey 1991; Richards 1995). Other measures of HRV obtained included HPM (the mean of the IBIs over the baseline period); HPSD (the mean of the S.D.s for the baseline period); HPFFT (the peak of the power spectrum in putative respiration frequency); Respfreg (the peak of the power spectrum in $\mathrm{Hz}$ ); and Hpprin (a principal component score). However, HPSSD has been the most often used measure of HRV (Richards 1995) and in the present study was the measure most consistently related to the heart rate conditioning variables.

\section{General conditioning apparatus and procedure}

Classical conditioning contingencies were controlled by a microcomputer, supplemented by Coulbourn TTL modules. All behavioral responses were recorded on a Grass Model 7 polygraph equipped with appropriate preamplifiers for each response. For data acquisition and analysis the amplifier outputs were connected to the computer where A-D conversion was performed in real time. Subjects were tested in a room separate from the room that contained the experimental apparatus. Signal generators, amplifiers, earphones, etc. were used to present the acoustic stimulus (tone) to the subjects. The airpuff was gated by a dc electric valve from a source pressurized to $2.5 \mathrm{psi}$. It was presented to the subject via a $0.5 \mathrm{~cm}$ diameter plastic tube connected to an adjustable head set (welder's goggles) that was mounted comfortably on the subject's head. The end of the tube was attached to a $2 \mathrm{~cm}$ piece of plexiglas mounted on the headset in front of the left eye (for more details see Durkin et al 1990). All stimulus events were controlled by a PC. Integrated EMG activity was assessed from the eye muscles, as previously described (Ayers et al 2003). This signal was input to the A-D converter of the computer, where it was sampled at $1000 \mathrm{~Hz}$.

The conditioning procedure used a compound CS with a 5 second light signal that co-terminated with a 1 second tone. The trial structure is diagrammatically presented in Figure 2. For one of the CSs (CS+), either a red or green light signal, the compound light-tone stimulus co-terminated with a 0.1 second corneal airpuff US. On the CS- trials the appropriate light/tone compound stimulus was presented, but was not followed by the airpuff US. The light signal color of a $\mathrm{CS}+$ trial was counterbalanced across subjects. Following a

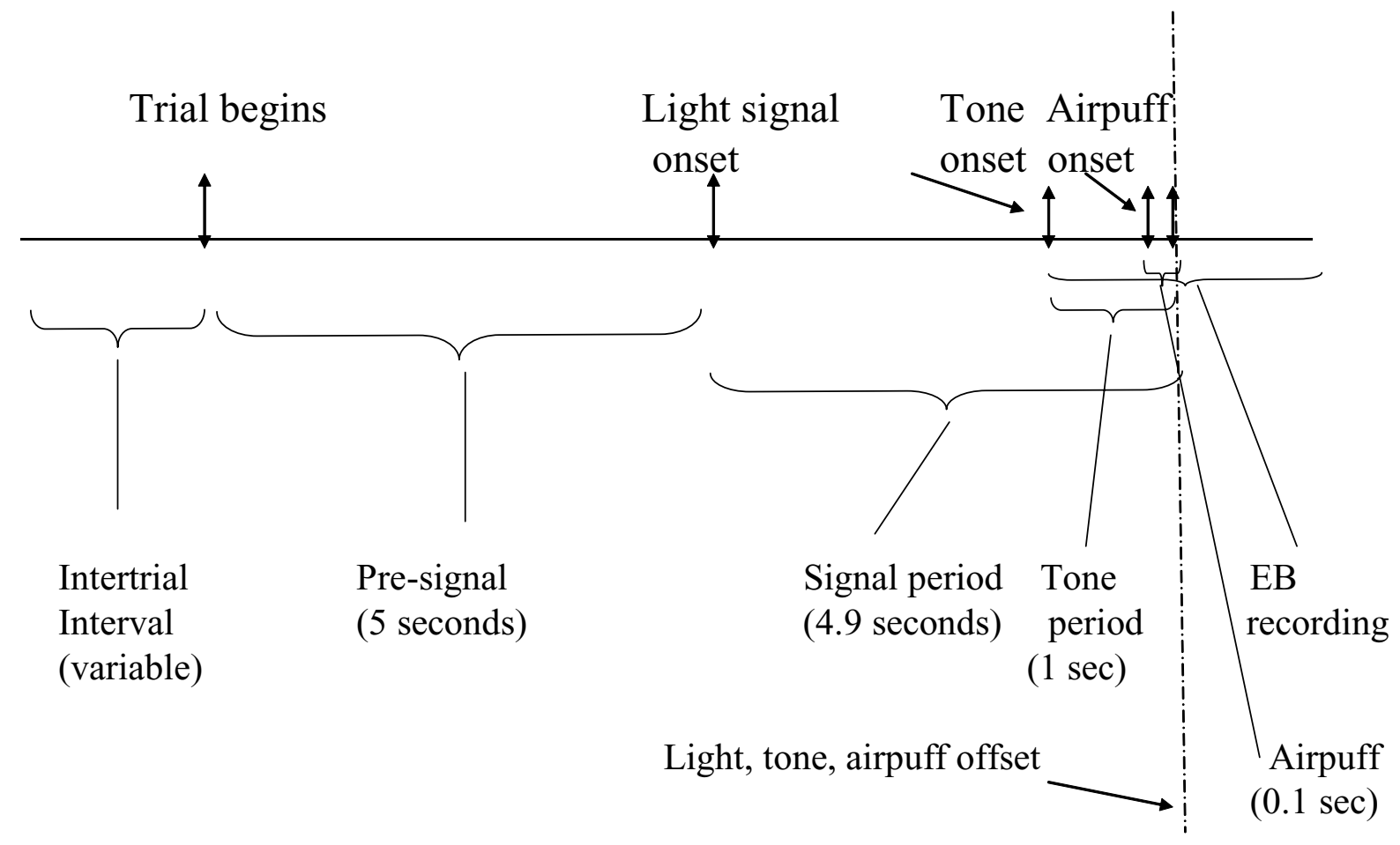

Figure 2. Structure of a conditioning trial. Conditioning trials that end with the airpuff unconditioned stimulus (US) are reinforced and designated CS+; trials that do not have the airpuff US are unreinforced and designated CS-. HR was measured during the 4.9 second signal period when the light was on before onset of the tone-corneal airpuff contngency, and compared with average HR of during the 5.0 second pre-signal baseline.

Note: Not drawn to time scale. 
variable intertrial interval of 10-20 seconds, the light signal, consisting of illumination of a PC screen, was presented for a period of 5 seconds. A 1 second, $1000 \mathrm{~Hz}, 75 \mathrm{db}$ tone was presented 4 seconds following onset of the light signal. The tone was followed by a 0.1 second, 2.5 psi corneal airpuff US on CS+ trials. Thus, the period of the light signal alone was 4.9 seconds (at which time the airpuff was presented). On CS - trials, the light and tone were presented but the corneal airpuff was omitted. Seventy-two mixed acquisition trials were given, half reinforced (ie, $\mathrm{CS}+$ ) and half nonreinforced (ie, CS-), presented in a pseudorandom order such that one type of CS did not occur three or more times in succession.

\section{Heart rate conditioning}

HR was measured continuously during the experiment and then analyzed for two periods of interest on each conditioning trial: the 5 second pre-light signal baseline period and the 4.9 second light signal period preceding the airpuff. The outcome HR conditioning variable was \% BPM change from trial pre-signal baseline HR during each 1.0 second interval of the signal period (the last interval was only 0.9 second). For each of the 72 trials, baseline HR was computed as the weighted mean heart period in BPM (Berntson et al 1995, 1997) of all IBI's during the 5 second period before onset of the light signal (ie,the last 5 seconds of the inter-trial interval). The HR for each signal interval was calculated as the weighted average of the beats that fell within that interval. The HR change score for each interval was calculated by subtracting pre-signal baseline HR from that signal interval $\mathrm{HR}$; the percent change score for each interval was then simply the quotient of HR change score over baseline HR $\mathrm{x}$ 100. Baseline HR on Trial 1 only was analyzed separately from the other trials, as an indicator of baseline HR when subjects were naïve to the paradigm.

\section{Eyeblink conditioning}

Details of measurement of EB conditioned responding are reported elsewhere (Ayers and Powell 2002; Ginsberg et al 2007).

\section{Results \\ Descriptives}

A total of 66 subjects (20 Combat PTSD+, 16 Combat PTSD-, and 30 Noncombat PTSD-) subjects completed the study, although only 49 subjects (16 Combat PTSD+, 12 Combat PTSD-, 21 Noncombat PTSD-) had valid heart rate recording data (Ginsberg et al 2007). The mean age of the 49 participants who completed the study was 52.3 (12.4) years, with no significant difference between subgroups $(F[2,46]=0.43, p>0.05$; Table 1$)$. There was no significant difference in education, sex, or racial composition between subgroups.

No subject was found to have had any diagnosis of psychosis or bipolar disorder (which were exclusion criteria), and only one subject had a notation of personality disorder. $30 \%$ of the sample had an indication of depression (not necessarily major depressive disorder), 21\% substance abuse/ dependence, and 6.7\% anxiety other than PTSD. However, the subgroups did not differ significantly in the incidence of these co-morbid mental diagnoses (Table 1).

The most prevalent prescription in our sample was for pain medication (56\%). Antidepressants were those found in VA drug class CN609, which is made up of selective serotonin reuptake inhibitors and serotonin-norepinephrine

Table I Descriptives of Combat PTSD+, Combat PTSD-, and Noncombat subgroups

\begin{tabular}{|c|c|c|c|c|c|}
\hline Variable & $\begin{array}{l}\text { Combat PTSD+ } \\
(n=16,32.7 \%)\end{array}$ & $\begin{array}{l}\text { Combat PTSD- } \\
(\mathrm{n}=12,24.5 \%)\end{array}$ & $\begin{array}{l}\text { Noncombat PTSD- } \\
(n=21,42.8 \%)\end{array}$ & $X^{2}(2, N=49)$ & $\mathbf{p}$ \\
\hline$\%$ Male & 75 & 83 & 81 & 2.33 & 0.33 \\
\hline \% Caucasian & 38 & 58 & 48 & 1.20 & 0.55 \\
\hline \% Depression & 44 & 33 & 19 & 1.82 & 0.42 \\
\hline$\%$ Other anxiety & 19 & 0 & 0 & 3.2 & 0.21 \\
\hline \multirow[t]{2}{*}{$\% \mathrm{Hx}$ substance } & 31 & 17 & 19 & 1.12 & 0.58 \\
\hline & & & & $F(2,46)$ & $\mathbf{p}^{\mathbf{a}}$ \\
\hline Age & $51.7(9.6)$ & $55.5(14.7)$ & $51.1(13.4)$ & 0.43 & 0.66 \\
\hline Education (years) & 13.5 (I.7) & $15.2(2.8)$ & $14.7(3.4)$ & 1.41 & 0.25 \\
\hline Mississippi Scale & I I9.I (24.2) & $77.3(21.8)$ & $67.4(18.7)$ & 28.13 & $<0.01$ \\
\hline Depression ${ }^{\mathrm{b}}$ & $60.2(11.2)$ & 49.I (15.5) & 44.9 (II.7) & 6.92 & $<0.01$ \\
\hline State anxiety ${ }^{c}$ & $48.1(10.7)$ & $39.1(12.0)$ & 32.4 (II.7) & 8.50 & $<0.01$ \\
\hline Trait anxietyc & $51.2(I I .5)$ & 39.0 (II.3) & $33.3(10.5)$ & 12.14 & $<0.01$ \\
\hline
\end{tabular}

Notes: aOmnibus F test; orthogonal contrasts all significant ( $\mathrm{p} \leq 0.02$ two-tailed, see Results); ${ }^{\mathrm{b}}$ Depression was measured as self-report on the Zung Depression Scale; ' ${ }^{\mathrm{C}} \mathrm{Ctate}$ and Trait Anxiety were measured as self-report on the Spielberger (1983) Anxiety Inventory, Form Y. 
reuptake inhibitors (eg, buproprion, citalopram, fluoxetine, mirtazapine, paroxetine, sertraline, trazodone, and vanlafaxine). These antidepressants were prescribed for $30 \%$ of the sample. Mood stabilizers (CN400; anticonvulsants prescribed at mood-stabilizing doses, such as carbamazepine and valproate) were prescribed for 10\%. Anxiolytics (CN302, benzodiazepine derivatives/hypnotics such as diazepam, lorazepam) were prescribed for 3\%. Current medication for hypertension was found to be prescribed for $53 \%$, for diabetes for $11 \%$, and beta-blockers and muscle relaxants both were prescribed for less than 5\%. Cross-tabulation of PTSD+ with psychotropic medication revealed that $44 \%$ of the subjects with PTSD (7/16) were not on any psychotropic medication at the time of the study.

\section{Psychometrics}

The three subgroups significantly differed on the psychometric test scores (Table 1), and post-hoc orthogonal comparisons revealed that all contrasts of (a) PTSD+ vs. PTSD- (Combat + Noncombat) were significant ( $\mathrm{p} \leq 0.01$ two-tailed) and (b) no contrast of Combat PTSD- vs. Noncombat PTSD- was significant ( $\mathrm{p} \geq 0.13$ two-tailed). The PTSD+ subgroup differed from the PTSD- subgroups, but the PTSD- subgroups did not differ from each other, thus forming a dichotomous classification of subjects into PTSD+ (Combat) and PTSD(Combat + Noncombat) subgroups.

\section{HRV and baseline HR}

The baseline pre-signal HR (see Trial Structure; Figure 2) on Trial 1 only, at which point subjects were naïve to the paradigm, was analyzed for differences between PTSD+/PTSDsubgroups. There was no significant difference in this measure (PTSD+ mean BPM = 71.7, SD = 7.3; PTSD- mean BPM $=70.1, \mathrm{SD}=10.2 ; \mathrm{t}[47]=-0.542, \mathrm{p}=0.59$ two-tailed). However, regression analysis revealed that Age was welldescribed by HRV and baseline BPM on Trial 1 (Table 2). The independence of these two predictor variables was shown by two indicators of multiple collinearity: a nonsignificant correlation $(\mathrm{r}[47]=-0.18, \mathrm{p}=0.29$ two-tailed $)$ and high tolerance $(0.968)$.
Table 2 Multiple linear regression equation for prediction of age using resting heart rate variability and heart rate (BPM) on Trial I $(\mathrm{n}=49)$

\begin{tabular}{lllll}
\hline Variable & B (SE) & Beta & T & Sig T \\
\hline Dependent variable:Age & & & & \\
HRV & $-0.9 \mathrm{I}(0.23)$ & $-0.5 \mathrm{I}$ & -3.97 & $<0.0 \mathrm{I}$ \\
Baseline HR (BPM) Trial I & $-0.53(0.17)$ & $-0.4 \mathrm{I}$ & -3.18 & $<0.0 \mathrm{I}$ \\
(Constant) & $100.04(12.4)$ & & 8.04 & $<0.0 \mathrm{I}$ \\
\hline
\end{tabular}

Note: Final equation: $R^{2}=0.35, F(2,46)=11.07, p<0.01$.

\section{Discriminative heart rate conditioning}

The percentage of BPM change during the 4.9 second light signal period was analyzed using a mixed-model ANOVA, with the between-subject factor of PTSD Group (+/-) and two within-subject (repeated measure) factors of CS Type (+/-) and Seconds of the signal period (5 intervals) (Table 3 ).

No interaction or main effect of CS Type was significant, indicating that HR did not demonstrate conditional discrimination between $\mathrm{CS}+$ and $\mathrm{CS}-$ signals. This result stands in contrast to EB, which did show conditional discrimination in this paradigm and is discussed further, below (Ginsberg et al 2007). The interaction of Seconds x PTSD was significant $(F[4,188]=2.6, p=0.04)$, and the main effect of Seconds was significant $(F[4,188]=7.4$, $p<0.01)$, but the main effect of PTSD was not significant $(p=0.20)$. Thus, PTSD appears to have an effect on the course of HR deceleration over the period of the light signal. The linear contrast of the PTSD $x$ Seconds interaction was $F(1,47)=5.2, p=0.07$, indicating a nearly significant difference between the slopes of the linear decline of $\%$ from baseline BPM for the PTSD+ vS. PTSD- subgroups when considered separately.

The Seconds $x$ PTSD significant interaction was further explored to isolate the source of the effect using (i) simple comparisons of subgroup differences in the intervals and (ii) simple effect of Seconds in PTSD+ and PTSD- subgroups separately.

(i) Inspection of Figure 3 in light of the significant interaction of PTSD $x$ Seconds in \% change both in BPM and linear trend suggested that the difference between the two subgroups was significant in some of the intervals. Simple comparisons of

Table 3 Mean change in \%BPM (SD) from the five second baseline pre-light signal period during five intervals of the 4.9 second light signal period for 72 eyeblink discriminative delay conditioning trials (combined across CS+ and CS- trials and across PTSD- and PTSD+ subgroups)

\begin{tabular}{lllll}
\hline Light signal period (seconds) & $0.0-0.99$ & $1.00-1.99$ & $2.00-2.99$ & $3.00-3.99$ \\
$\%$ BPM change from 5 second pre-signal & $-0.18(.68)$ & $-0.44(.7 \mathrm{I})$ & $-0.54(.97)$ & $-0.68(1.07)$ \\
baseline period (all trials) $(\mathrm{n}=49)$ & & & $-0.92(\mathrm{I}) .25)$ \\
\hline
\end{tabular}



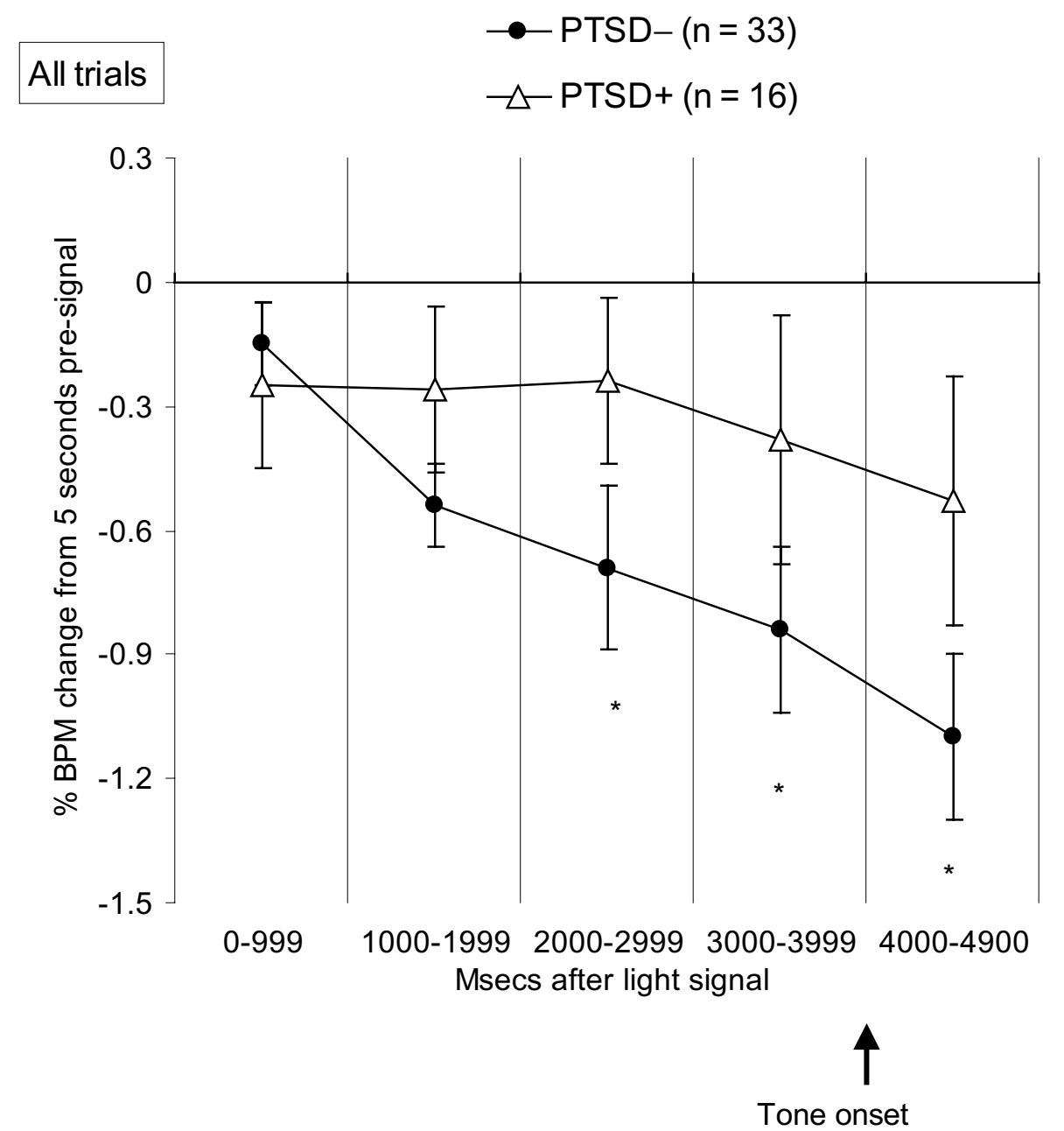

Figure 3 Mean change in \%BPM $( \pm$ I SEM) from 5 second pre-signal baseline for I second intervals during the 4.9 second signal period for PTSD+ and PTSD- subgroups separately, averaged across CS+ and CS- trials. The interaction of Seconds $x$ PTSD and the main effect of Seconds were significant ( $P<0.04$ and $p<0.01$, respectively). The linear trend of the PTSD $\times$ Seconds interaction was nearly significant $(P=0.07)$, indicating a nearly significant difference between the slopes of the HR change for the PTSD+ vs. PTSD- subgroups when considered separately. $\mid \%$ change in BPM| in the PTSD+ subgroup was lower than $\mid \%$ change in BPM| in PTSD- (P < 0.05 , one-tailed) during the third, fourth, and fifth intervals.

Notes: ${ }^{*}|\mathrm{PTSD}+|<|\mathrm{PTSD}-|(\mathrm{p}<0.05$, one-tailed $)$.

the subgroup means at each interval, using the mean square of the original analysis as error term (Keppel 1982), revealed a significant difference $(|\mathrm{PTSD}+|<\mid$ PTSD $-\mid, \mathrm{p}<0.05)$ at the third, fourth, and fifth intervals.

(ii) The test of the simple main effect of Seconds in the PTSD subgroups separately, again using the mean square of the original analysis as error term (Keppel 1982), revealed that the effect of Seconds was significant in the PTSDsubgroup and the downward slope was linear (both p's $<0.01$ ) (Figure 4a). Furthermore, HR in the PTSDsubgroup decelerated (ie, the change from baseline was significantly less than zero) on CS- and CS+ trials in all of the signal intervals after the first $(p \geq 0.14$ in the first interval, $\leq 0.01$ in second through fifth intervals, all two-tailed); the difference in \%BPM change between
CS- vs. CS+ trials was not significant in any interval (all p's $\geq 0.11$, one-tailed). In contrast, however, there was no longer a significant effect of Seconds or linear slope in the PTSD+ subgroup (Figure 4b). HR in the PTSD+ subgroup decelerated from baseline on CS- trials in the third through fifth intervals ( $p \leq 0.04$, two-tailed), but was not significantly different from baseline on CS+ trials in any of the five signal intervals (all p's $\geq 0.36$, two-tailed); the $\mid \% \mathrm{BPM}$ change| on $\mathrm{CS}+$ trials was significantly lower than that of CS- trials in the third interval ( $p<0.04$, one-tailed).

\section{Discussion}

When we reported reduced EB responses to an aversive US (airpuff) in PTSD+ combat veterans, we speculated that the 
(a) PTSD- veterans.

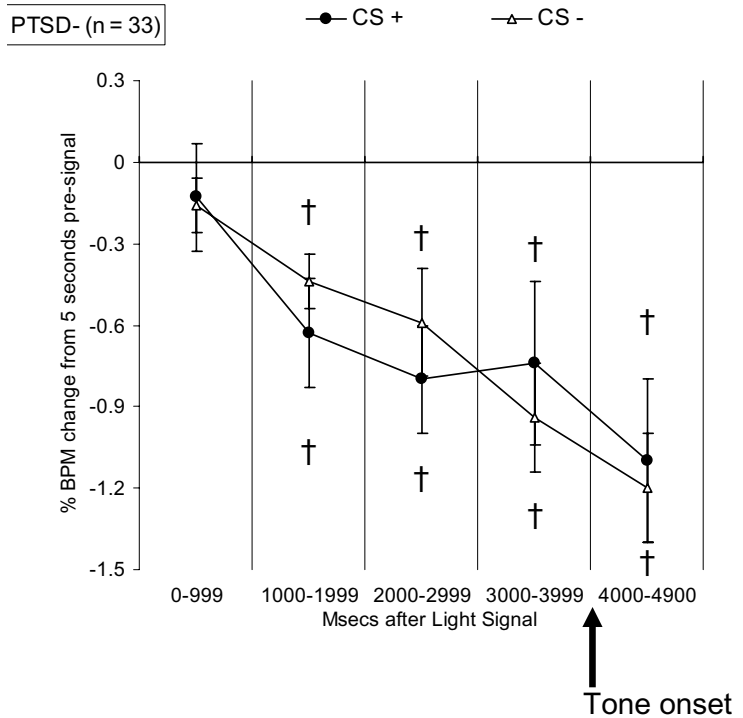

(b) PTSD+ veterans

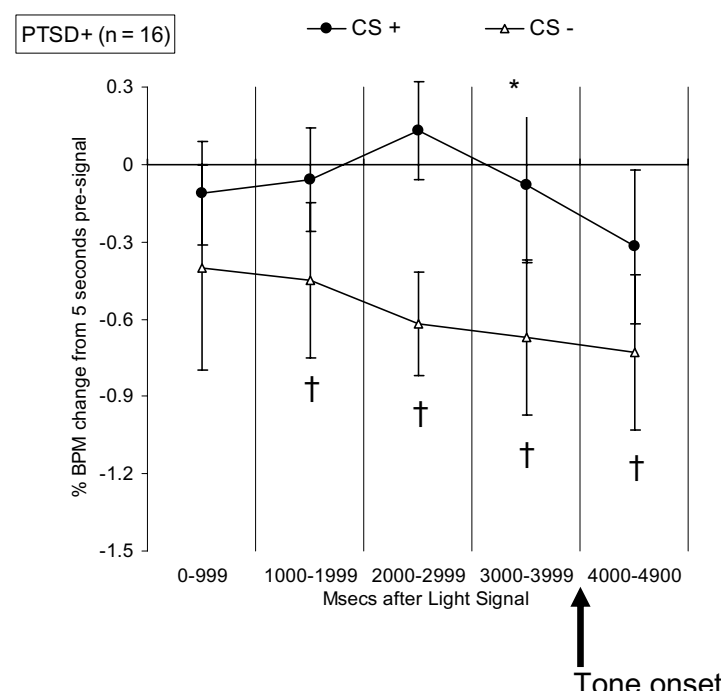

Figure 4 Mean change in \%BPM ( \pm I SEM) from 5 second pre-signal baseline for I second intervals during the 4.9 second signal period for 36 CS+ and 36 CS- trials. (a) Veterans without PTSD (PTSD-). Simple main effect of Seconds was significant and the downward slope was linear in the PTSD- subgroup; HR decelerated (was less than zero) on CS- and CS+ trials in all intervals after the first; there was no significant difference between CS- and CS+ trials in any interval. (b) Veterans with PTSD (PTSD+). In contrast to PTSD- veterans, the simple main effect of Seconds and linear trend were not significant in the PTSD+ subgroup; HR decelerated on CS- trials in the third through fifth intervals but did not significantly change from baseline in any interval on CS+ trials; HR deceleration on CS- trials was significantly greater than on CS+ trials during the third interval. Notes: ${ }^{\dagger} \mathrm{HR}$ deceleration (\% BPM change significantly less than zero); ${ }^{*}|\mathrm{CS}-|>|\mathrm{CS}+|(\mathrm{p}=0.04$, one-tailed).

hyporesponsivity was due to the presence of a signal that perfectly predicted onset of the aversive stimulation (Ginsberg et al 2007; Powell 2007). This finding would be consistent with studies using the startle reflex among human adults with anxiety, which indicate that predictability of aversive stimulation results in lowered conditionability compared to aversive stimulation that is unpredictable (Grillon 2002a, $2002 b$ ). Associative learning of the tone-shock contingency appeared to be disrupted, and habituation of the defensive reflex accelerated, because the $\mathrm{CS}+$ light signal conferred complete predictability of the aversive US onto the delay discrimination conditioning task. The present results show that the same PTSD+ veterans who showed EB hyporesponsivity also have the normal post-CS deceleration of HR to the CS+ eliminated during the signal period preceding the aversive stimulation. Thus, autonomic conditioning (to the warning signal) and somatomotor conditioning (to actual aversive stimulation) are both reduced in PTSD+ combat veterans in $\mathrm{CS}+$ trials, when the signal perfectly predicts aversive stimulation. Decreased cardiac responding is thus clearly an aspect of PTSD.

A question that arises, however, is whether the observed bradycardia to both CS types in PTSD- controls and to CS- only in PTSD+ is an OR, rather than a CR. The observed bradycardia occurred over the entire 4.9 second period of the light signal and monotonically increased (ie, constant rate of HR deceleration), with a linear decreasing slope. If the observed bradycardia was simply a nonsignal OR, it should have habituated (Sokolov et al 2002). If the observed bradycardia is a signal OR, then, along with the subsequent contingent bradycardic response, it is part of the entire bradycardic CR. Thus, the HR change over the 4.9 second signal period observed in the present study appears to be a CS (light)-elicited bradycardiac response to the signal properties of the light-tone contingency.

The main finding of the study is that HR conditioning to the CS+ signal was reduced in veterans with PTSD. The data did not indicate that conditioned HR discrimination took place in the PTSD- controls, although as noted above, EB did differentiate CS+/CS in all subgroups (Ginsberg et al 2007). Discriminative conditional EB responding was due to the US (airpuff) contingency to the tone component of the light-tone compound $\mathrm{CS}+$, which occurred in the last $0.1 \mathrm{sec}$ of the 5 second signal period. The HR response discussed in the present article was, however, based on the 4.9 sec light-tone CS before onset of the US. We would argue that, among the controls, the discriminative properties of the light-tone compound signal were not as powerful as those of 
the tone-shock reinforcement contingency, and so the $\mathrm{CS}+/$ CS- discrimination was not observed for HR conditioning (light-tone pairing) alone but did occur in eyeblink conditioning (tone-shock). Sokolov (2002) suggested that the ORs to conditioning signals without high discriminability "are intensified for more precise analysis, in order to make decisions about whether they are conditioned stimuli or not." (p. 34). Because the signal period was relatively long, development of a signal OR to both CS types was enabled and no $\mathrm{CS}+/ \mathrm{CS}-$ autonomic differentiation was seen in the PTSD- controls. The situation is different in the PTSD+ subgroup, however, where the signal OR does not appear by the second interval (1-2 seconds, and the subsequent monotonic deceleration associated with the contingency response never develops. It is interesting to note that this lab has previously reported that interference with the decelerative HR CR may adversely affect acquisition of the EB CR in the rabbit (Powell 1979).

Differential HR conditioning as a function of post-CS interbeat intervals can be shown in rabbits, for example using periorbital shock as the CS+ reinforcement and never reinforcing the CS-, as a function of post-CS interbeat intervals (Powell and Ginsberg 2005). In contrast, and of special interest, is the finding of a study of human subjects by Flaten (1998), which used apparatus and stimulus parameters very similar to those of the present study. Experimentally induced high arousal ( $4 \mathrm{mg} / \mathrm{kg}$ oral caffeine) produced differential CS+/CS- EB conditioning but $\mathrm{CS}+/ \mathrm{CS}$ - differentiation by HR did not occur, even though the first and second HR decelerative components (D1 and D2 respectively) both increased-indicating orienting (the D1 component) and contingent (D2) HR decelerative responding to $\mathrm{CS}+/ \mathrm{US}$ pairings (Flaten 1998). These results seem to be compatible with those of the present study.

Furthermore, the finding that CS- elicited bradycardia was not significantly different for $\mathrm{CS}+$ versus $\mathrm{CS}-$ for the HR response in the control subjects, whereas this difference was significant for the EB response. It thus may be related to the different brain substrates for somatomotor and autonomic responses, and thus merely a reflection of additional differences between conditioning of nonspecific versus specific responses. Autonomic conditioned responding (as indexed by HR) and somatomotor conditioned responding (as indexed by EB) can be doubly dissociated, reflecting the different central nervous system (CNS) substrates that mediate them (Lavond et al 1984; Powell 1999, 2007; Powell et al 2000; Thompson 2000). These results are interpretable in terms of different neural substrates underlying HR vs. EB conditioning (Figure 5).

Classically conditioned HR responding has a different CNS substrate from conditioned EB responses (Powell 1999, 2007). Corticolimbic structures including the medial prefrontal cortex (mPFC) and amygdale central nucleus (ACN) are critical for the development of classically conditioned changes in HR to stimuli (Powell et al 2000), while the essential CNS substrates for EB conditioning involve the deep nuclei of the cerebellum (Thompson 2000). As noted above, there is a double dissociation of the CNS substrates of EB and HR classically conditioned responses; damage to cerebellar structures does not affect HR conditioning (Lavond et al 1984), while damage to corticolimbic structures that mediate HR conditioning do not affect either unconditioned HR responding or EB conditioning under optimal conditions (Buchanan and Powell 1982a, 1982b).

The neural substrate of conditioned HR adjustments incorporates the $\mathrm{mPFC}$ and the $\mathrm{ACN}$, and their interconnections (Powell et al 1997), both of which also provide separate direct projections to visceral control nuclei in the medulla (Buchanan et al 1994). The role of hippocampal inputs to $\mathrm{mPFC}$ during autonomic conditioning may also be relevant under some conditions, such as when the CS is a constant or contextualized environmental factor and the US is fear-producing (eg, using freezing as the dependent variable), rather than a defensive reflex as in the present case. The cerebellar vermis is also known to participate in conditioned bradycardia (Supple and Kapp 1990), but the relationship of cerebellar learned autonomic adjustments to similar control by the mPFC and amygdala is not clear.

Nonetheless, there is ample data from controlled animal experimental studies to support the conclusion that dorsal regions of $\mathrm{mPFC}$ (areas 24 and 32) are critical in the acquisition of conditioned nonspecific cardiac inhibitory adjustment, but are not involved in unconditioned cardiac responding to stimulation (Buchanan and Powell 1982a). Kapp and colleagues (1992) have similarly shown that lesions centered on the ACN greatly attenuate but do not completely abolish HR conditioning in the rabbit, and neuronal activity in these structures is associated with that stage of conditioning during which autonomic CRs are normally acquired. Analysis of single neuron activity in mPFC during HR conditioning using both adrenergic and cholinergic peripheral blockade showed no change compared to the same cells during vehicle administration, a result that indicates the neuronal activity is centrally initiated in the $\mathrm{mPFC}$ and not the result of peripheral afferent feedback 


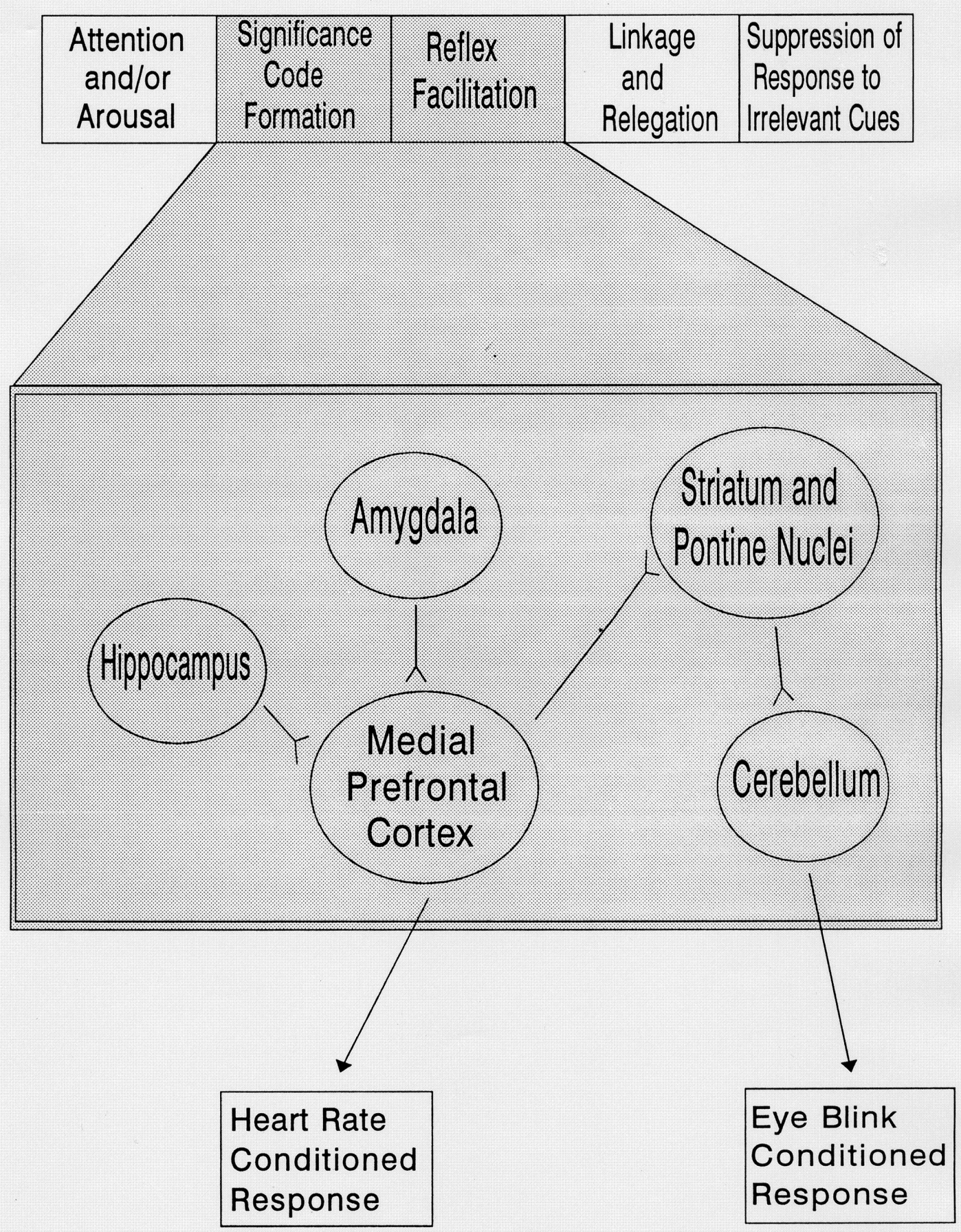

Figure 5 Diagram of known pathways between corticolimbic structures mediating autonomic consitioning such as heart rate and extrapyramidal structures that normally participate in somatomotor conditioning such as eyeblink (Powell 1999).

(Powell and Ginsberg 2005). Because the above manipulations do not affect concomitant somatomotor (eg, EB) conditioning, the $\mathrm{mPFC}$ and $\mathrm{ACN}$ appear to constitute the central component of the neural substrate upon which nonspecific inhibitory HR adjustments are classically conditioned.
In summary, our data indicate that conditioned HR responding (deceleration) is impaired in PTSD. These findings may be related to CNS circuits which are known to be involved in conditioned physiological regulation of the heart. Because conditioned physiological regulation of the heart is 
mediated primarily by $\mathrm{mPFC}$ and the amygdala, these findings indicate dysfunction of these structures, and may be further related to other problems that often develop in combat veterans with with PTSD. Such an explanation is compatible with the widespread belief that alterations in $\mathrm{mPFC}$-amygdala connectivity is a component of PTSD. For example, the attention, learning, and memory impairments in PTSD are consistent with frontal lobe dysfunction (eg, Golier and Yehuda 2002), and decreased $\mathrm{mPFC}$ and anterior cingulate cortex activation along with hyperactivity in the amygdala during exposure to trauma-related stimuli haves been reported in PTSD (Lanius et al 2001; Shin et al 2001; Bremner et al 2008). Dysregulation of the mPFC-amygdala axis would also be expected to contribute to other problems that are commonly observed to develop in combat veterans with PTSD, such as impulsivity, depression, and poor judgment.

\section{References}

Ayers ED, Powell DA. 2002. Multiple response measures during classical conditioning. J Neurosci Methods, 14:33-8.

Ayers ED, White J, Powell DA. 2003. Pavlovian eyeblink conditioning in combat veterans with and without post-traumatic stress disorder. Integr Physiol Behav Sci, 38:230-47.

Berntson GG, Bigger JT, Eckberg DL, et al. 1997. Heart rate variability: Origins, methods, and interpretive caveats. Psychophysiology, 34:623-48.

Berntson GG, Cacioppo JT, Quigley KS. 1995. The metrics of cardiac chronotropism: Biometric perspectives. Psychophysiology, 32:162-71.

Blake DD, Weathers FW, Nagy LM, et al. 2000. Instruction manual for Clinician Administered PTSD Scale (CAPS), Appendix 1. Edn. White River Junction, VT: National Center for Post-traumatic Stress Disorder.

Bremner JD, Elzinga B, Schmahl C, et al. 2008. Structural and functional plasticity of the human brain in posttraumatic stress disorder. Prog Brain Res, 167:171-86.

Buchanan S, Thompson R, Maxwell B, et al. 1994. Efferent connections of the medial prefrontal cortex in the rabbit. Exp Brain Res, 100:469-83.

Buchanan SL, Powell DA. 1982a. Cingulate cortex: Its role in Pavlovian conditioning. J Comp Physiol Psychol, 96:755-74.

Buchanan SL, Powell DA. 1982b. Cingulate damage attenuates conditioned bradycardia. Neurosci Lett 29:261-8.

Burriss LH, Ayers ED, Ginsberg JP, et al. 2007. Learning and memory impairment in PTSD: Relationship to depression. Depress Anxiety, 25:149-57.

Durkin M, Prescott L, Jonet CJ, et al. 1990. Photoresistive measurement of the Pavlovian conditioned eyelid response in human subjects. Psychophysiology, 27:599-603.

Flaten MA. 1998. Caffeine-induced arousal modulates somatomotor and autonomic differential classical conditioning in humans. Psychopharmacology (Berl), 135:82-92.

Gatchel RJ, Lang PJ. 1973. Accuracy of psychophysical judgments and physiological response amplitude. J Exp Psychol, 98:175-83.

Ginsberg JP, Ayers ED, Burriss L, et al. 2006. A study of the association between retrospective appraisal of childhood reactivity and post-discharge traumatic stress in combat veterans. Traumatology, 12:61-82.

Ginsberg JP, Ayers ED, Burriss LH, et al. 2007. Discriminative delay Pavlovian eyeblink conditioning in veterans with and without Posttraumatic Stress Disorder. J Anxiety Disord, Aug 30 [Epub ahead of print].

Golier J, Yehuda R. 2002. Neuropsychological processes in post-traumatic stress disorder. Psychiatr Clin North Am, 25:295-315.
Grillon C. 2002a. Associative learning deficits increase symptoms of anxiety in humans. Biol Psychiatry, 51:851-8.

Grillon C. 2002b. Startle reactivity and anxiety disorders: Aversive conditioning, context, and neurobiology. Biol Psychiatry, 52:958-75.

Kapp B, Whalen P, Supple W, et al. 1992. Amygdaloid contributions to conditioned arousal and sennsory information processing. In: Aggleton JP (Ed.). The Amygdala: Neurobiological Aspects of Emotion, Memory, and Mental Dysfunction. New York, NY: Wiley-Liss, Inc., pp. 229-54.

Keane TM, Cadell JM, Taylor KL. 1988. Mississippi Scale for combatrelated posttraumatic stress disorder: Three studies in reliability and validity. J Consult Clin Psychol, 56:85-90.

Keppel G. 1982. Design and Analysis: A Researcher's Handbook. Englewood Cliffs, NJ: Prentice-Hall, Inc.

Lanius RA, Williamson PC, Densmore M, et al. 2001. Neural correlates of traumatic memories in posttraumatic stress disorder. Am J Psychiatry, $158: 1920-2$

Lavond D, Lincoln J, McCormick D, et al. 1984. Effect of bilateral lesions of the dentate and interpositus cerebellar nuclei on conditioning of heart-rate and nictating membrane/eyelid responses in the rabbit. Brain Res, 305:323-30.

Maxwell B, Powell DA, Buchanan SL. 1994. Multiple and single unit activity in area 32 (prelimbic region) of the medial prefrontal cortex during Pavlovian heart rate conditioning in the rabbit. Cereb Cortex, 4:230-46.

McFall ME, Smith DE, Mackay PW, et al. 1990. Reliability and validity of Mississippi Scale for combat-related Post-traumatic Stress Disorder. Psycholl Assess, 2:114-21.

Ohman A. 1988. Nonconscious control of autonomic responses: A role for Pavlovian conditioning? Biol Psychol, 27:113-35.

Orr SP, Metzger L, Lasko N, et al. 2000. De novo conditioning in trauma-exposed individuals with and without posttraumatic stress disorder. J Abnorm Psychol, 109:290-8.

Peri T, Ben-Shakhar G, Orr S, et al. 2000. Psychophysiologic assessment of aversive conditioning in posttraumatic stress disorder. Biol Psychiatry, 47:513-19.

Pitman RK. 1989. Post-traumatic stress disorder, hormones, and memory. Biol Psychiatry, 26:221-3.

Pitman RK, Delahanty D. 2005. Conceptually driven pharmacologic approaches to acute trauma. CNS Spectrums, 10:99-105.

Powell DA. 1979. Peripheral and central muscarinic cholinergic blockade: Effects on Pavlovian conditioning. Bull Psychonom Soc, 14:161-4.

Powell DA. 1999. A behavioral stages model of classical (Pavlovian) conditioning: application to cognitive aging. Neurosci Biobehav Rev, 23:797-816

Powell DA. 2007. Medial prefrontal cortex and Pavlovian conditioning Cogn Sci 2:127-74

Powell DA, Buchanan SL, Gibbs CM 1990. Role of the prefrontal-thalamic axis in classical conditioning. In: Uylings HBM, Eden CGV, Bruin JPCD, et al. (Eds.). The Prefrontal Cortex: Its Structure, Function, and Pathology. Amsterdam, The Netherlands: Elsevier Science Publishers BV, pp. 433-66.

Powell DA, Chachich M, Murphy V, et al. 1997. Amygdala-prefrontal interactions and conditioned bradycardia in the rabbit. Behav Neurosci, 111:1056-74.

Powell DA, Ginsberg JP. 2005. Single unit activity in the medial prefrontal cortex during Pavlovian heart rate conditioning: Effects of peripheral autonomic blockade. Neurobiol Learn Mem, 84:200-13.

Powell DA, McLaughlin J, Chachich M. 2000. Classical conditioning of autonomic and somatomotor responses and their central nervous system substrates. In: Woodruff-Pak DS, Steinmetz JE (Eds). Eyeblink Classical Conditioning: Animal Models. Boston, MA: Kluwer Academic Publishers, pp. 257-86.

Richards JE. 1995. Reliability of respiratory sinus arrhythmia in R-R intervals in 14, 20, and 26 week old infants. Infant Behav Dev, 18:155-61.

Richards JE, Casey BJ. 1991. Heart rate variability during attention phases in young infants. Psychophysiology, 28:43-53. 
Shin LM, Whalen PJ, Pitman RK, et al. 2001. An fMRI study of anterior cignulate function in posttruamatic stress disorder. Biol Psychiatry, 50:932-42.

Sokolov EN, Spinks JA, Naatanen R, et al. 2002. The Orienting Response in Information Processing. Mahwah, New Jersey: Lawrence Erlbaum Associates.

Spielberger CD, Gorsuch RL, Lushene R, et al. 1983. Manual for the State-Trait Anxiety Inventory (Form Y). Palo Alto, CA: Mind Garden.
Supple Jr WF, Kapp BS. 1990. The anterior cerebellar vermis: Essential involvment in classically conditioned bradycardia in the rabbit. Behav Neurosci, 104:3705-11.

Thompson RF. 2000. Discovering the brain substrates of eyeblink classical conditioning. In: Steinmetz JE, Woodruff-Pak DS (Ed). Eyeblink Classical Conditioning Volume 2: Animal Models. Boston, MA: Kluwer Academic Publishers, pp. 17-49.

Zung WWK. 1965. A self rating depression scale. Arch Gen Psychiatry, $12: 63-70$ 